\title{
The surgical outcomes of aortic valve replacement in patients with aortic valve lesions caused by Behcet's disease: lessons we learned
}

\author{
Xiaoning Sun ${ }^{1,2}$, Li Yuan ${ }^{1,2}$, Junjiang Liu ${ }^{1,2}$, Quanlin Yang ${ }^{1,2}$, Huan Liu ${ }^{1,2}$, Hongqiang Zhang ${ }^{1,2}$, \\ Chunsheng Wang ${ }^{1,2}$
}

${ }^{1}$ Department of Cardiac Surgery, Zhongshan Hospital, Fudan University, Shanghai, China; ${ }^{2}$ Shanghai Institute of Cardiovascular Disease, Shanghai, China

Contributions: (I) Conception and design: X Sun, C Wang; (II) Administrative support: C Wang, H Zhang; (III) Provision of study materials or patients: L Yuan, H Liu; (IV) Collection and assembly of data: X Sun, J Liu; (V) Data analysis and interpretation: X Sun; (VI) Manuscript writing: All authors; (VII) Final approval of manuscript: All authors.

Correspondence to: Hongqiang Zhang, MD. Department of Cardiac Surgery, Zhongshan Hospital, Fudan University, and Shanghai Institute of Cardiovascular Disease, Shanghai 200032, China. Email: zhang.hongqiang@zs-hospital.sh.cn; Chunsheng Wang, MD. Department of Cardiac Surgery, Zhongshan Hospital Fudan University, and Shanghai Institute of Cardiovascular Disease, Shanghai 200032, China. Email: wang.chunsheng@zs-hospital.sh.cn.

Background: For Behcet's disease patients with aortic root lesions, the disease often manifests as aortic valve regurgitation (AR). Following aortic valve replacement (AVR), many of these patients often suffer perivalvular leakage and valve dehiscence, requiring a second or third operation. In this study, we report the outcomes of 20 patients who underwent AVR to manage aortic root lesions caused by Behcet's disease, and the lessons we learned.

Methods: From October 2013 to September 2019, a total of 50 patients with Behcet's disease underwent AVR at our institution. Among them, isolated AVR was performed in 15 preoperatively undiagnosed cases. The other 5 patients were preoperatively diagnosed and underwent modified AVR. All patients were contacted for a follow-up. Valve function was evaluated using echocardiography.

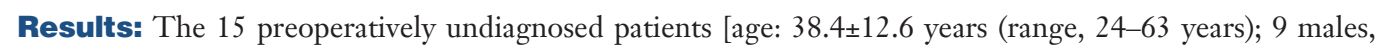
6 females] underwent isolated AVR as their primary procedure. Echocardiography revealed that valve dehiscence occurred in $13(86.7 \%)$ patients postoperatively after a mean interval of $10.8 \pm 8.4$ months. These patients accepted a second operation, and 1 of them accepted a third operation because of a pseudoaneurysm of the distal anastomosis site. For the other 5 patients [age: 38.8 \pm 9.5 years (range, 27-55 years); 4 males, 1 female] who underwent modified AVR, neither AR nor prosthetic valve detachment were observed during the echocardiography follow-up, and none required a secondary operation.

Conclusions: For behcet's disease patients with AR, there was a high rate of valve dehiscence after isolated AVR. When compared with traditional AVR, we found that modified AVR was the optimal choice for patients who received standardized preoperative treatment.

Keywords: Behçet's disease; aortic root disease; aortic valve replacement (AVR)

Submitted Oct 19, 2021. Accepted for publication Oct 29, 2021.

doi: $10.21037 /$ atm-21-5673

View this article at: https://dx.doi.org/10.21037/atm-21-5673 


\section{Introduction}

Behcet's disease (BD) is a chronic inflammatory disease involving multiple systems. The etiology is unknown and is characterized by recurrent oral and genital ulcers, uveitis, and erythema nodosa $(1,2)$. Aortic root disease caused by $\mathrm{BD}$ is one of the main causes of death in $\mathrm{BD}$ patients and often manifests as aortic valve regurgitation (AR) and ulcerative defects in the aortic root or myocardium. The incidence and nature of cardiac involvement in Behçet's disease are not yet clearly documented. The mechanism of BD causing aortic root disease is not clear, and it may be related to vasculitis, aortic valve prolapse and perforation. The most effective treatment method is cardiac surgery, supplemented by a variety of immunomodulatory drugs and hormone therapy. Due to the special pathogenesis of BD, the aortic root tissue of $\mathrm{BD}$ patients is fragile, and most patients undergoing simple aortic valve replacement (AVR) surgery often have paravalvular leaks (PVL), requiring a second or even third surgery $(3,4)$.

At present, due to the small number of cases and the significant individual differences in BD patients with aortic root disease, there is still no standard surgical method to treat the condition. Many surgeons have sought to modify the surgery for the purpose of improving its efficacy. For example, Chen (5) used the modified Bentall technique, in which the valved conduit was proximally attached to the left ventricular outflow tract instead of the fragile annulus to prevent prosthetic valve detachment. Another study by Ando and Liang used Teflon felt outside the aortic root to prevent tearing of the anastomosis (6-8). A further study by Takashi (9) described using a sandwich reinforcement on the aortic root with Teflon felt. They placed a ring in the subannular position as a reinforcement. they sandwiched the aortic annulus between the subannular ring and the prosthetic valve. However, in some of these tests, the sample size was too small to confirm the results, and in some of them the clinical results were not ideal. In the past, not enough was known about BD and subsequently it was not clearly diagnosed prior to operation. However, in recent years, with an improved understanding of $\mathrm{BD}$, new approaches have emerged. In our study, we performed isolated AVR on $15 \mathrm{BD}$ patients with $\mathrm{AR}$, and modified AVR (8) with reinforcement of the aortic root on a further 5 $\mathrm{BD}$ patients with $\mathrm{AR}$. Interrupted mattress stitches from the outside in and horizontally through the aortic wall in the same plane below the valve ring in a circumferential fashion is the different from Takashi's methods. Here we report the follow-up results and the lessons we learned. We present the following article in accordance with the STROBE reporting checklist (available at https://dx.doi.org/10.21037/atm-215673).

\section{Methods}

\section{Patients}

From October 2013 to September 2019, a total of 50 BD patients underwent aortic root surgery in our hospital. Among them, 20 patients who required isolated AVR surgery were enrolled in our study. Clinical data of these patients were manually accrued retrospectively from their electronic medical records and hospital charts. During hospitalization, we also collected the general information of each patient (including gender, age) and other various items by reviewing each patient's record. We also checked their individual test results (including preoperative and postoperative cardiac ultrasonography, aortic computed tomography angiography (CTA), pathology report) and medical history data. Their mean age was $38.4 \pm 6.9$ years (range, 21-55 years) at the time of the first operation. At our hospital, eleven patients underwent the first operation, and nine patients were transferred from other hospitals after their initial isolated AVR procedure. We selected 5 patients who were preoperatively diagnosed with aortic root disease caused by BD to undergo modified AVR. In addition, echocardiography revealed that these 5 patients had severe AR. All procedures performed in this study involving human participants were in accordance with the Declaration of Helsinki (as revised in 2013). The study was approved by ethics board of Zhongshan Hospital, Fudan University, Shanghai, China (No. B2021-675R). Individual consent for this retrospective analysis was waived.

\section{BD diagnosis (10)}

Patients were diagnosed with BD based upon clinical symptoms, laboratory examination, intraoperative exploration, and pathological examination of the aortic valve and aortic wall, and in accordance with the revised diagnostic criteria outlined in the International Criteria for Behcet's Disease (ICBD) published by the International Study Group (ISG). Aortic valve regurgitation or paravalvular leakage is determined by transthoracic ultrasound cardiogram or transesophageal echocardiography. 


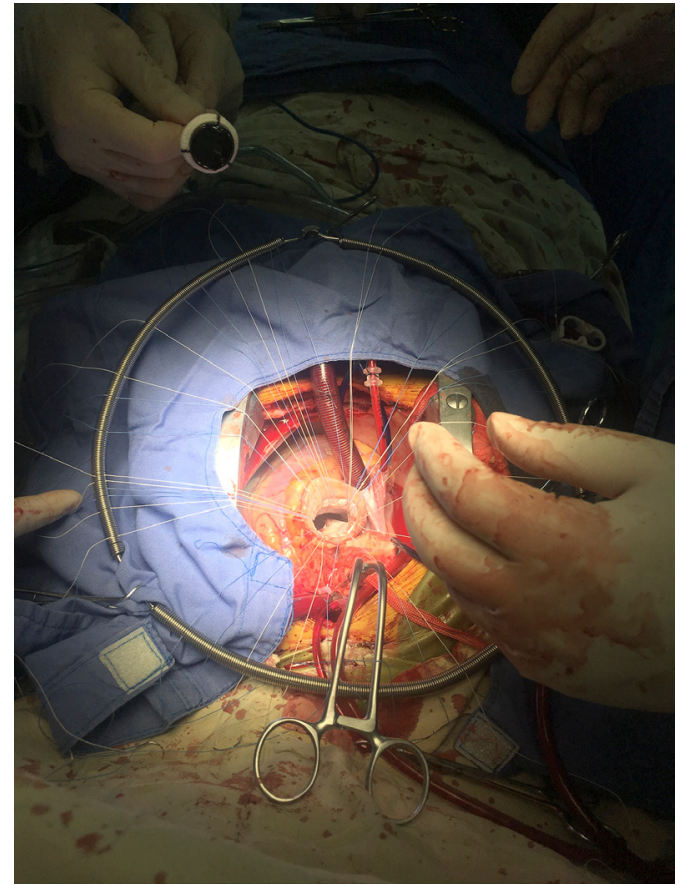

Figure 1 Beneath the ostia of both coronary arteries, interrupted mattress stitches are used from the outside in and horizontally through the aortic wall in the same plane below the valve ring in a circumferential fashion.

\section{Operative technique}

Patients without aortic root destruction received standard AVR with either a mechanical or stented tissue valve inserted using interrupted or pledgeted mattress sutures.

\section{Modified AVR}

After the establishment of cardiopulmonary bypass, blood cardioplegia was our preferred method of myocardial protection. Above the sinus-tublar junction, transect the ascending aorta to explore the aortic wall and valve leaflets, after resection of the leaflets, the aortic root was externally mobilized to a level immediately below the nadir of the aortic annulus. The diameter of the prosthesis was then calculated. The diameter was measured by the insertion of a mechanical valve sizer. Then, the modified aortic valve replacement surgery was performed. We separated the aortic root to the level of the valve annulus as it is essential to perform reinforcement on the aortic root when replacing the prosthetic valve. Beneath the ostia of both coronary arteries, we used interrupted mattress stitches from the outside in and horizontally through the aortic wall in the same plane below the valve ring in a circumferential fashion. It was then anchored in the aortic wall. Finally, the sewing ring of the prosthetic valve was done sequentially (Figure 1). The aortic wall was sandwiched between the outer cushion and the sewing ring of the artificial valve. Then, the incision of the ascending aorta was anastomosed. reinforcing the sewing ring on the inner side of the posterior wall and the outer side of the anterior wall by suturing discontinuously and fixing with felt. Caution was taken when suturing the same horizontal to make sure the tension was balanced.

\section{Immunosuppressive therapy}

After the operation, patients entered the cardiac surgery intensive care unit for observation. All patients were required to take anticoagulant drugs for the rest of their lives and regularly undergo a follow-up for blood coagulation function [adjusting the anticoagulant dose according to the international rate (INR)]. After admission to the intensive care unit, immunosuppressive therapy was started. methylprednisolone ( $40 \mathrm{mg} /$ day) was given intravenously first, followed by prednisolone $(10 \mathrm{mg} /$ day $)$ and thalidomide $(50 \mathrm{mg} /$ day $)$ orally.

\section{Follow-up}

All patients were contacted for a follow-up. In the outpatient clinic, transthoracic color Doppler echocardiography is used to evaluate the shape and function of the heart and valves. Records about complications such as infection, embolism, and bleeding. Recent outpatient visits and hospitalization records were checked through the electronic medical record system.

\section{Statistical analysis}

Continuous variables are expressed as mean \pm standard error of the mean. Student's $t$ test was used for comparison of continuous variables between the groups. All data management and analyses were performed using STATA 11.0 software (Stata Corp, College Station, TX, USA). A P value of 0.05 was considered statistically significant

\section{Results}

The mean follow-up duration for the 20 patients after primary surgery was 15-120 months (median: 43 months). 
Table 1 Outcome of isolated AVR

\begin{tabular}{|c|c|c|c|c|c|}
\hline Gender & Age & First operation & $\begin{array}{c}\text { Paravalvular leakage time after } \\
\text { operation (month) }\end{array}$ & Second Operation & Pseudoaneurysm \\
\hline M & 21 & AVR & / & / & / \\
\hline $\mathrm{F}$ & 63 & AVR & / & / & I \\
\hline $\mathrm{F}$ & 26 & AVR & 14 & Bentall & Yes \\
\hline M & 25 & AVR & 5 & Bentall & / \\
\hline M & 50 & AVR & 12 & Bentall & / \\
\hline M & 39 & AVR & 10 & Bentall & / \\
\hline M & 34 & AVR & 7 & Bentall + CABG & Yes \\
\hline $\mathrm{F}$ & 30 & AVR & 12 & Bentall & / \\
\hline $\mathrm{F}$ & 54 & $\mathrm{AVR}+\mathrm{MVP}$ & 6 & Bentall & / \\
\hline$M$ & 50 & AVR & 13 & Modified AVR & / \\
\hline
\end{tabular}

AVR, aortic valve replacement; CABG, coronary artery bypass grafting; ECMO, extracorporeal membrane oxygenation.

For the 15 preoperatively undiagnosed patients (age: $38.4 \pm 12.6$ years (range, 24-63 years); 9 males, 6 females) who underwent isolated AVR as the primary procedure, trans-thoracic color Doppler echocardiography revealed that paravalvular leak occurred in $13(86.7 \%)$ patients postoperatively after a mean interval of $10.8 \pm 8.4$ months (range, 3-36 months). All these patients accepted a second operation. During the follow-up after the second operation (which included redoing modified AVR in 1 case and traditional AVR in 10 (Bentall or Bentall + CABG), there were 2 deaths. One death occurred due to right ventricular failure after reoperation with root reconstruction. The other death occurred due to right coronary artery damage during a sternal reentry. Despite having a coronary artery bypass, multiple organ failure occurred in this patient after transient extracorporeal membrane oxygenation support. During the follow-up after the second operation, 1 patient also accepted to undergo a third operation because of a pseudoaneurysm of the distal anastomosis site (Table 1).

\section{Outcome of modified AVR}

For the 5 patients [age: $38.8 \pm 9.5$ years (range, $27-55$ years);
4 males, 1 female] who underwent modified AVR, the time of each patient's preoperative medical treatment ranged from 2 to 5 years and was mainly constituted by a glucocorticoid and immunosuppressive agent. Before surgery, some related routine preoperative examinations were needed to ensure each patient's erythrocyte sedimentation rate (ESR) and C-reactive protein (CRP) were stable and normal.

Following the primary procedure of modified AVR, no deaths occurred in these 5 patients. Furthermore, neither AR nor paravalvular leak were observed during the followup. These patients remained symptom-free with no obvious abnormality in heart function. The data of all patients who underwent modified AVR in our hospital can be seen in Table 2.

\section{Comments}

BD with aortic disease often occurs in young patients, and is often not accompanied by other underlying diseases such as rheumatic fever, hypertension, and coronary atherosclerosis. The morphological changes are prolapse, valve leaflet perforation, and tear (11). The functional changes are mainly valve insufficiency, followed by aseptic 
Table 2 The follow-up: AVR vs. modified AVR

\begin{tabular}{lcc}
\hline Characteristics & $\begin{array}{c}\text { Modified AVR } \\
(\mathrm{n}=5)\end{array}$ & AVR $(\mathrm{n}=15)$ \\
\hline Age & $38.8 \pm 9.5$ & $38.4 \pm 12.6$ \\
Gender (male/female) & $4 / 1$ & $9 / 6$ \\
Diagnosed/undiagnosed & $6 / 0$ & $0 / 15$ \\
Paravalvular leakage & 0 & $13 / 15$ \\
Pseudoaneurysm & 0 & 2 \\
Multiple operations & 0 & $13 / 15$ \\
Death & 0 & 2 \\
\hline
\end{tabular}

AVR, aortic valve replacement.

vegetation, which needs to be differentiated from infective endocarditis (IE) (12). Cardiac surgeons generally have a lack of knowledge and treatment experience around $\mathrm{BD}$ as it is often confused with other valvular heart diseases.

Due to the peculiarities of the pathogenesis of $\mathrm{BD}$, when performing an isolated AVR, PVL is the most common complication after AVR. This is because the prosthetic valve is often detached from the natural valve annulus during reoperation $(13,14)$.

In Ando's reports (6), the incidence of paravalvular leak in patients after isolated AVR were $66.7 \%$. Another study (14) reported that valve dehiscence or periprosthetic leakage requiring reoperation occurred in $78.9 \%$ of patients (15/19). In this study, for patients undiagnosed preoperatively, PVL occurred in 10 patients (87.7\%). We also found that in our study as well as others, aortic root or ascending aorta replacement after a prior AVR resulted in a higher morbidity and mortality rate $(15,16)$.

Why does PVL occur after isolated AVR? A reasonable speculation is that the artificial valve is placed in the enlarged annulus and the tension is therefore greater, resulting in a certain tension on the fragile inflammatory annulus, and therefore making it easier for PVL or even valve avulsion to occur. Aortic root replacement removes the diseased aortic sinus and part of the ascending aorta and the tensions generated when the mechanical valve leaflet is opened and closed is dispersed on the artificial blood vessel. Thereby, the tension on the natural valve ring is correspondingly reduced, as well as the chances of PVL. On the other hand, the aortic valve annulus is arc-shaped, and the suture position is also arranged along the arc-shaped annulus. After AVR, the artificial aortic valve is forced to turn the arc into a plane, and part of the suture will generate upward tension. In this way, the suture does not penetrate the full thickness of the aortic wall. Under the action of tension, the inflammatory tissue of $\mathrm{BD}$ is brittle and prone to avulsion, causing PVL.

To solve these problems, modified total aortic root replacement (5) and the Modified Bentall procedure (16) have been tried by some surgeons. For example, Ando and Liang put Teflon felt outside the aortic root to prevent tearing of the anastomosis (6-8). However, the sample size of this and other previous studies were too small to confirm their efficacy, and in some of them the clinical results were not ideal.

Based on the same hypothesis, our institution made some modifications to the AVR procedure. For example, we only performed modified AVR on patients who had been definitely diagnosed with aortic root disease associated with $\mathrm{BD}$, our suture line was stitched from the outside in through the felt strip, and the operation took place on the aortic wall on the level of annulus for the purpose of making the tension on the same horizontal to avoid the formation of shear and to prevent the annulus from tearing. By so doing, the tension was dispersed on the felt rather than the annulus. During the operation, we also paid particular attention to the coronary arteries (where the stitches remain) to prevent injury. From our follow-up results with these $5 \mathrm{BD}$ patients, we obtained satisfactory results, with no PVL yet to be found. Since the patients we selected had a clear preoperative diagnosis for $\mathrm{BD}$, and the state of their illness was stable, the physical condition of the patients was good. This means that our results may be relatively limited, and that the treatment effect for patients with severe aortic root disease needs to be carefully evaluated.

It must be emphasized that $\mathrm{BD}$ is a chronic multisystemic inflammatory disorder of unknown etiology, Glucocorticoids and various immunosuppressive drugs have been shown to be necessary for controlling inflammation and improve the surgical results of patients with aortic root disease caused by BD. In the case of a clear diagnosis of BD at first operation, isolated AVR should be avoided, aortic root replacement operation or modified AVR should be performed at the operation.

\section{Conclusions}

Preoperative diagnosis is extremely important for determining the type of operation BD patients should undergo and is critical for determining the effects of surgery $(11,12)$. Even today in small hospitals, some 
isolated AVR procedures are performed. For this reason, it is very important to strengthen the understanding of the pathological changes of the aortic root in BD patients, and to choose the appropriate surgical method to reduce complications. For patients with BD involving aortic root disease, preoperative diagnosis of the primary disease is very important. In our study, the incidence of valve dehiscence in patients after isolated AVR were very high. To conclude, for BD patients who receive effective treatment for the primary disease, do not display any obvious abnormalities in the ascending aorta, we feel modified AVR is the ideal choice.

\section{Limitations}

Our study had certain limitations, including the fact that only some of the aortic root lesions caused by BD required surgical treatment, and that the number of patients in the study was quite small. Our experience may therefore not fully represent the full appearance of the disease. However, it may enrich the current literature on BD.

\section{Acknowledgments}

Funding: The authors acknowledge funding from the National Natural Science Foundation of China (No. 81970442).

\section{Footnote}

Reporting Checklist: The authors have completed the STROBE reporting checklist. Available at https://dx.doi. org/10.21037/atm-21-5673

Data Sharing Statement: Available at https://dx.doi. org/10.21037/atm-21-5673

Conflicts of Interest: All authors have completed the ICMJE uniform disclosure form (available at https://dx.doi. org/10.21037/atm-21-5673). The authors have no conflicts of interest to declare.

Ethical Statement: The authors are accountable for all aspects of the work in ensuring that questions related to the accuracy or integrity of any part of the work are appropriately investigated and resolved. All procedures performed in this study involving human participants were in accordance with the Declaration of Helsinki (as revised in 2013). The study was approved by ethics board of Zhongshan Hospital, Fudan University, Shanghai, China (No. B2021-675R). Individual consent for this retrospective analysis was waived.

Open Access Statement: This is an Open Access article distributed in accordance with the Creative Commons Attribution-NonCommercial-NoDerivs 4.0 International License (CC BY-NC-ND 4.0), which permits the noncommercial replication and distribution of the article with the strict proviso that no changes or edits are made and the original work is properly cited (including links to both the formal publication through the relevant DOI and the license). See: https://creativecommons.org/licenses/by-nc-nd/4.0/.

\section{References}

1. Sharma S, Pandey NN, Sinha M, et al. Etiology, Diagnosis and Management of Aortitis. Cardiovasc Intervent Radiol 2020;43:1821-36.

2. Hatemi G, Seyahi E, Fresko I, et al. One year in review 2020: Behçet's syndrome. Clin Exp Rheumatol 2020;38 Suppl 127:3-10.

3. Ma WG, Zheng J, Zhu JM, et al. Aortic regurgitation caused by Behçet's disease: surgical experience during an 11-year period. J Card Surg 2012;27:39-44.

4. Ghang B, Kim JB, Jung SH, et al. Surgical Outcomes in Behcet's Disease Patients With Severe Aortic Regurgitation. Ann Thorac Surg 2019;107:1188-94.

5. Chen LW, Wu XJ, Cao H, et al. Valved Conduit Attached to Left Ventricular Outflow Tract for Valve Detachment in Behçet's Disease. Ann Thorac Surg 2017;103:e301-3.

6. Ando M, Kosakai Y, Okita Y, et al. Surgical treatment of Behçet's disease involving aortic regurgitation. Ann Thorac Surg 1999;68:2136-40.

7. Ando M, Sasako Y, Okita $Y$, et al. Valved conduit operation for aortic regurgitation associated with Behçet's disease. Jpn J Thorac Cardiovasc Surg 2000;48:424-7.

8. Liang MY, Yao JP, Zhang X, et al. Surgical technique for aortic regurgitation attributable to Behcet's disease: modified aortic valve replacement with reinforcement of the aortic wall. Eur J Cardiothorac Surg 2012;41:647-8.

9. Azuma T, Yamazaki K, Saito S, et al. Aortic valve replacement in Behcet's disease: surgical modification to prevent valve detachment. Eur J Cardiothorac Surg 2009;36:771-2.

10. International Team for the Revision of the International Criteria for Behçet's Disease (ITR-ICBD). The International Criteria for Behçet's Disease (ICBD): a 
collaborative study of 27 countries on the sensitivity and specificity of the new criteria. J Eur Acad Dermatol Venereol 2014;28:338-47.

11. Karadag O, Bolek EC. Management of Behcet's syndrome. Rheumatology (Oxford) 2020;59:iii108-17.

12. Han JK, Kim HK, Kim YJ, et al. Behçet's disease as a frequently unrecognized cause of aortic regurgitation: suggestive and misleading echocardiography findings. J Am Soc Echocardiogr 2009;22:1269-74.

13. Jeong DS, Kim KH, Kim JS, et al. Long-term experience of surgical treatment for aortic regurgitation attributable

Cite this article as: Sun X, Yuan L, Liu J, Yang Q, Liu H, Zhang H, Wang C. The surgical outcomes of aortic valve replacement in patients with aortic valve lesions caused by Behcet's disease: lessons we learned. Ann Transl Med 2021;9(20):1607. doi: 10.21037/atm-21-5673 to Behçet's disease. Ann Thorac Surg 2009;87:1775-82.

14. Marzban M, Mandegar MH, Karimi A, et al. Cardiac and great vessel involvement in "Behcet's disease". J Card Surg 2008;23:765-8.

15. Embrey RP. Modified Cabrol's technique for composite replacement of the aortic valve and ascending aorta. J Card Surg 1993;8:562-6.

16. Dun Y, Shi Y, Guo H, et al. Outcome of reoperative aortic root or ascending aorta replacement after prior aortic valve replacement. J Thorac Dis 2021;13:1531-42. 